\title{
Caso Clínico Pediátrico para diagnóstico
}

\author{
ALBERTO VIDAL G.*, KARLA MOËNNE B.**, \\ PATRICIO RODRÍGUEZ D.*** y GUSTAVO CALDERÓN A.****
}

\section{Historia clínica}

Escolar de 7 años 7 meses, refiere dolor torácico ocasional tipo puntada. En anamnesis dirigida, se obtiene antecedente de tos y dificultad respiratoria recurrente, ha sido tratado con inhalador y antibióticos en varias oportunidades en el último año.

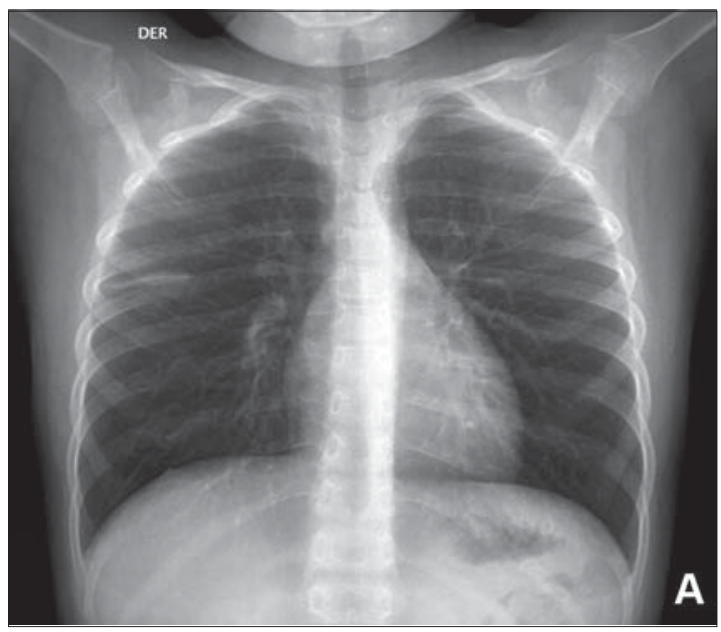

Aporta el antecedente que 8 meses antes chocó contra un ventanal, cayendo sobre los fragmentos de vidrio. Presentó lesiones perforantes de abdomen que requirieron cirugía y extracción de un trozo de vidrio del hígado, con buena evolución posterior.

Se indica radiografía de tórax AP y lateral (Figura 1).

Figura 1. Rx tórax, A) Frontal y B) lateral.

\section{¿Cuál sería su hipótesis diagnóstica y conducta?}

1) Atelectasia subsegmentaria.

2) Cuerpo extraño.

3) Derrame intercisural.

4) Otro.

* Pediatra, Becado Broncopulmonar Infantil, Universidad de Chile.

** Radióloga infantil, Clínica Las Condes.

*** Cirujano de Tórax, Clínica Las Condes.

**** Radiólogo, Centro Radiológico Copiapó. 
Bion 3 Nuevo 


\section{Hallazgos radiológicos}

La radiografía AP (Figura 1a) evidencia una imagen lineal de densidad de partes blandas en proyección de la cisura menor, que en la radiografía lateral (Figura 1b) se representa como una pequeña imagen redondeada, de la misma densidad, bien delimitada, proyectada a nivel suprahiliar.

Con la hipótesis de atelectasia subsegmentaria, se indicó kinesioterapia respiratoria y control radiológico, que no mostró variación de la imagen radiográfica. Se realizó tomografía computada (TC) de tórax con contraste.
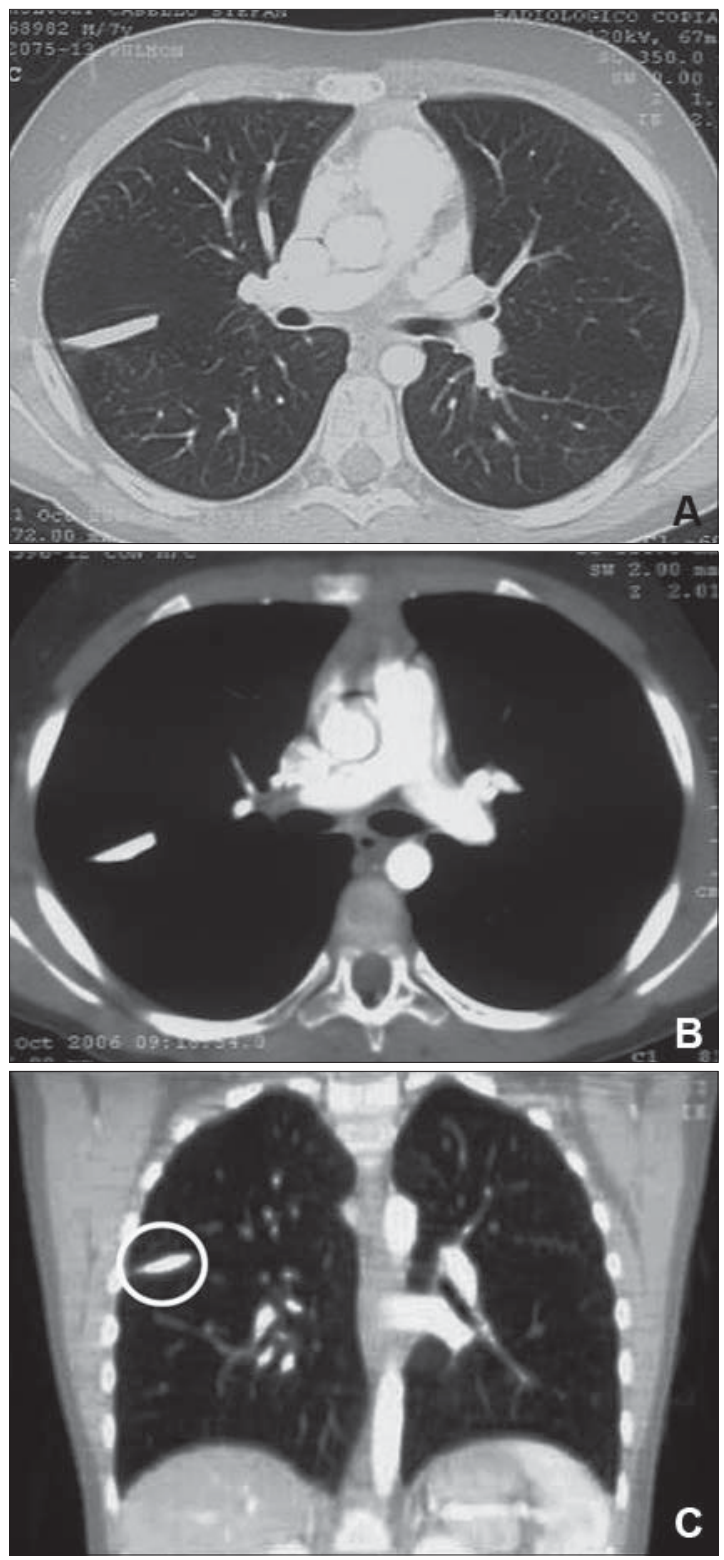

Figura 2. Tomografía computada de tórax con contraste, a) Corte axial, ventana pulmonar; b) Corte axial, ventana mediastínica; c) Reconstrucción coronal.
La TC de tórax confirmó la presencia de cuerpo extraño a nivel del tercio medio del hemitórax derecho (Figuras 2 y 3). El paciente se trasladó a un centro de mayor complejidad, donde se realizó en forma electiva, videotoracoscopía que demostró cuerpo extraño en el espacio pleural derecho, a nivel de la cisura menor. Se efectuó sin dificultad la extracción de un trozo de vidrio de 25 x 5 x $3 \mathrm{~mm}$ (Figura 4). Se dejó drenaje pleural y el paciente evolucionó sin complicaciones.

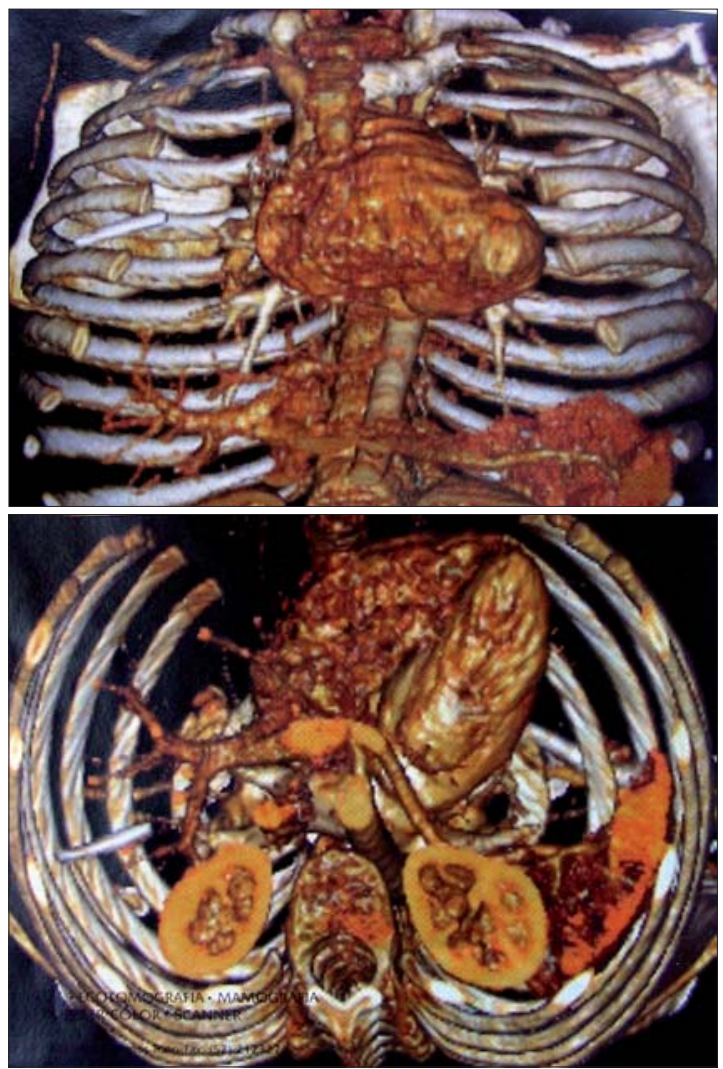

Figura 3. Reconstrucciones volumétricas de TC a) Coronal: b) Axial céfalo-caudal.

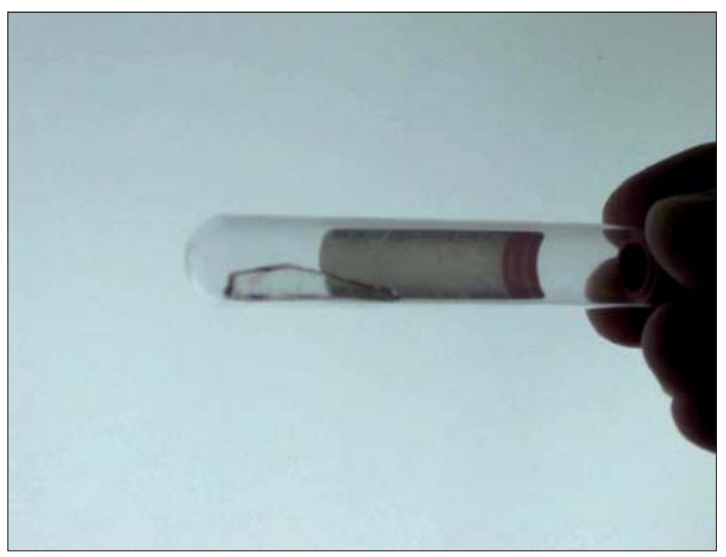

Figura 4. Fragmento de vidrio extraído por videotoracoscopía. 


\section{Discusión}

En relación al caso reportado, es importante discutir el diagnóstico diferencial de la imagen radiológica. Si bien en la radiografía AP la imagen lineal podría hacer sospechar una atelectasia o menos probablemente líquido intercisural, la radiografía lateral plantea la duda en relación a estos diagnósticos, por el escaso diámetro de la lesión en esa proyección. En el caso del derrame intercisural, también llamado tumor evanescente en adultos, existe un signo clásico de cambio de configuración en las diversas proyecciones, típicamente aspecto de "limón" en la placa lateral ${ }^{1}$. Desafortunadamente, con frecuencia no se dispone de mayores antecedentes al interpretar las radiografías de tórax, ya que el antecedente clínico de traumatismo y el aspecto descrito en la proyección lateral, permitirían plantear la posibilidad de presencia de un cuerpo extraño retenido, como se comprobó en la tomografía computada de tórax (Figura 2).

El trauma torácico es la segunda causa de muerte por trauma en pediatría. Las lesiones torácicas ocurren en el 5\% de los niños hospitalizados por trauma. Tienen diversa severidad y comprometen estructuras desde la pared torácica hasta los órganos mediastínicos; la mayoría se manifiestan en las primeras horas posteriores al trauma ${ }^{2}$. Existe un pequeño porcentaje de lesiones residuales o crónicas atribuibles al trauma, entre las que se cuentan los cuerpos extraños retenidos en la cavidad torácica.

Las complicaciones relacionadas con los cuerpos extraños torácicos no son nuevas. En la era preantibiótica de la tuberculosis pulmonar, se describía el plombaje, tratamiento que consistía en depositar cuerpos extraños de diferentes características en la cavidad torácica para inducir el colapso de las cavernas de los pacientes adultos. Este tipo de tratamiento, que no se utiliza en la actualidad, ha provocado complicaciones tardías relacionadas con la migración de los cuerpos extraños, que han requerido tratamiento videotoracoscópico ${ }^{3}$.

La literatura menciona en forma anecdótica algunas complicaciones crónicas en relación a traumatismos torácicos penetrantes, que agrupan a la mayoría de los casos reportados. Entre ellas destacan el empiema pleural y los cuerpos extraños pleurales retenidos, generalmente elementos metálicos y proyectiles en hombres jóvenes víctimas de agresiones o accidentes ${ }^{4-7}$.

Una publicación reciente comunicó una serie de 22 casos, con pacientes entre 2 y 73 años, recopilados durante 30 años, que presentaron cuerpos extraños residuales en pleura y pared torácica ${ }^{8}$. Los mecanismos implicados fueron traumáticos, ya sea accidentales o iatrogénicos. En esta revisión, los casos pediátricos fueron infrecuentes y correspondieron a causas iatrogénicas (procedimientos broncoscópicos) y a lesiones accidentales, que también se han descrito en la tercera edad ${ }^{9}$.

Si bien se han reportado extracciones videotoracoscópicas de vidrios en corazón y mediastino, éstos son elementos muy infrecuentes entre los materiales extraídos después de un trauma torácico ${ }^{10,11}$.

En los últimos años ha aumentado el uso de videotoracoscopía en el trauma torácico. Está especialmente recomendada en manejo de colecciones pleurales infectadas, neumotórax persistente, lesiones mediastínicas, lesiones pericárdicas y en evaluación del diafragma en pacientes con trauma tóracoabdominal penetrante; requiere hemodinamia estable ${ }^{12,13}$. La videotoracoscopía es el tratamiento ideal para este tipo de exploraciones en los pacientes pediátricos.

\section{Bibliografía}

1.- FRASER R, MÜLLER N, COLMAN N, PARÉ P. Diagnosis of Diseases of the Chest. W.B. Saunders Company, Philadelphia, Pennsylvania, USA, 1999.

2.- ALTERMAN D, DALEY B, KENNEDY A, RAJU R, LEE S. Considerations in pediatric trauma. E-medicine [Sitio en Internet]. Hallado en: http://www. emedicine.com/med/topic3223.htm. Acceso el $21 \mathrm{de}$ agosto de 2009. [Links].

3.- WEISSBERG D, WEISSBERG-KASAV D. Late complications of collapse therapy for pulmonary tuberculosis. Chest 2001; 120: 847-51.

4.- SUDAN R, PRASAD S, RAVIKUMAR R, STANLEY

J. Empyema caused by an unusual retained pleural foreign body. Indian J Thorac Cardiovasc Surg 1991; 7: 50-1.

5.- MARSICO G, ALMEIDA A, AZEVEDO D, VENTURINI G, AZEVEDO A, MARSICO PDOS S. Video-assisted thoracoscopic removal of foreign bodies from the pleural cavity. J Bras Pneumol 2008; 34: 241-4

6.- LIU Y, LIU H, LIN P, CHANG C. Thoracoscopic retrieval of foreign body after penetrating chest injury: report of two cases. Changgeng Yi Xue Za Zhi 1999; 22: $117-22$

7.- WILLIAMS C, HAUT E, OUYANG H, RIALL T, MAKARY M, EFRON D, et al. Video-assisted thoracic surgery removal of foreign bodies after penetrating chest trauma. J Am Coll Surg 2006; 202: 848-52.

8.- WEISSBERG D, WEISSBERG-KASAV D. Foreign 
bodies in pleura and chest wall. Ann Thorac Surg 2008; 86: 958-61.

9.- EDIL B, TRACHTE A, KNOTT-CRAIG C, ALBRECHT R. Video-assisted thoracoscopic retrieval of an intrapleural foreign body after penetrating chest trauma. J Trauma 2007; 63: E5-6.

10.- BARTEK J, GRASCH A, HAZELRIGG S. Thoracoscopic retrieval of foreign bodies after penetrating chest trauma. Ann Thorac Surg 1997;
63: $1783-85$.

11.- YANG X, SHEN X. A piece of glass in the heart. Ann Thorac Surg 2006; 81: 335-6.

12.- AHMED N, JONES D. Video-assisted thoracic surgery: state of the art in trauma care. Injury 2004; 35: 47989.

13.- CASÓS S, RICHARDSON J. Role of thoracoscopy in acute management of chest injury. Curr Opin Crit Care 2006; 12: 584-9.

Correspondencia a:

Dr. Alberto Vidal G.

Dirección: Holanda 87, Dpto. 604, Providencia, Santiago

E-mail: aevgmd@yahoo.es 